University of Nebraska - Lincoln

DigitalCommons@University of Nebraska - Lincoln

Faculty Publications: Materials Research

Science and Engineering Center

Materials Research Science and Engineering

Center

2005

\title{
Multiscale Phenomena in Bruggeman Composites
}

\author{
Ralph Skomski \\ University of Nebraska at Lincoln, rskomski2@unl.edu \\ Jiangyu Li \\ University of Nebraska-Lincoln, jjli@uw.edu \\ Jian Zhou \\ University of Nebraska-Lincoln, jzhou@unlserve.unl.edu \\ David J. Sellmyer \\ University of Nebraska-Lincoln, dsellmyer@unl.edu
}

Follow this and additional works at: https://digitalcommons.unl.edu/mrsecfacpubs

Part of the Materials Science and Engineering Commons

Skomski, Ralph; Li, Jiangyu; Zhou, Jian; and Sellmyer, David J., "Multiscale Phenomena in Bruggeman Composites" (2005). Faculty Publications: Materials Research Science and Engineering Center. 86. https://digitalcommons.unl.edu/mrsecfacpubs/86

This Article is brought to you for free and open access by the Materials Research Science and Engineering Center at DigitalCommons@University of Nebraska - Lincoln. It has been accepted for inclusion in Faculty Publications: Materials Research Science and Engineering Center by an authorized administrator of DigitalCommons@University of Nebraska - Lincoln. 


\title{
Multiscale Phenomena in Bruggeman Composites
}

\author{
Ralph Skomski, Jiangyu Li, * Jian Zhou, and David J. Sellmyer \\ Department of Physics and Astronomy and Center for Materials Research and Analysis, \\ *Department of Engineering Mechanics and Center for Materials Research and Analysis \\ University of Nebraska, Lincoln, NE 68588
}

\begin{abstract}
Mechanical, magnetic, and transport properties of arbitrary inhomogeneous composites are investigated by a Bruggeman-type mean-field approach. The theory yields materials parameters as functions of the volume fractions, geometries, and materials constants of the phases. Each system is described by a single response parameter g, which is equal to the percolation threshold of the composite. For macroscopic systems, the approach yields very simple expressions, but nanoscale and multiferroic effects yield relatively complicated corrections to $\mathrm{g}$. In the respective cases, the parameter $g$ depends on the length scale of the composite and has the character of a combination of magnetic, electric, and mechanical degrees of freedom.
\end{abstract}

\section{INTRODUCTION}

Composites combine the advantages of different single-phase materials and range from naturally occurring biological structures and traditional materials to artificial materials used in transport, space, microelectronic, and other high-tech applications [1-11]. For example, the composite structure of naturally occurring skeletal materials, such as bones and wood, ensures stiffness without brittleness, and the same principle is exploited in artificial mechanical materials, from concrete to reinforced polymers.

The question arises how the properties of a composite depend on the properties of the constituents and on how the length scale and geometry of the structure. Bruggeman's early work on the effective-medium description of dielectric and other composites [1] has lead to the concept of an effective medium. The idea is to start from exact solutions for small volume fractions of a second phase in a main or matrix phase. Arbitrary volume fractions are treated by selfconsistently embedding the phases in an effective medium [1]. Figure 1 shows some geometries.

The approach describes a variety of static and dynamic electromagnetic, mechanical, and diffuse phenomena. Physical phenomena include but are not restricted to electrical conduction (conducting composites, insulating inclusions in metallic matrix, metalsuperconductor composites), thermal conduction (heat insulation using composite construction materials), diffusion (hydrogen and nitrogen transport in intermetallic composites), magnetism (effective susceptibility), electrodynamics (static and dynamic dielectric response of inhomogeneous media, materials with negative index of refraction), rheology (viscosity of colloidal suspensions, such as blood, food, gels), mechanical composites (elasticity of reinforced construction materials, filled polymers, such as car tires). 


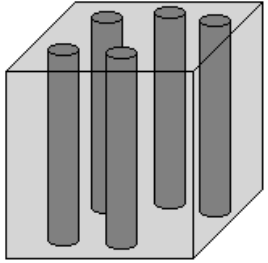

(a)

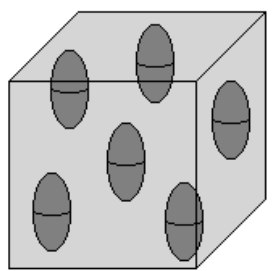

(d)

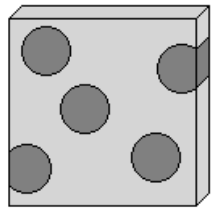

(g)

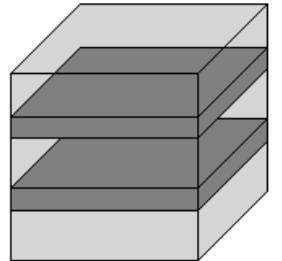

(b)

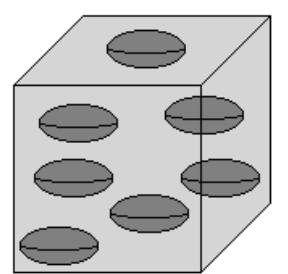

(e)

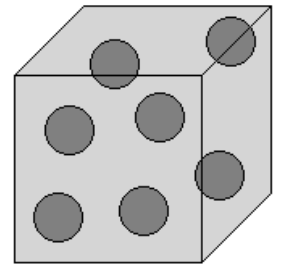

(c)

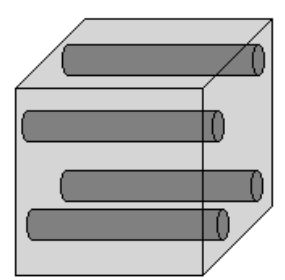

(f)

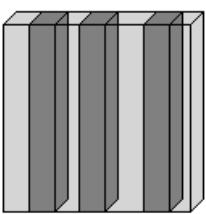

(h)

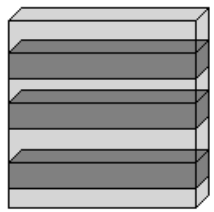

(j)

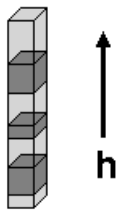

(k)

Figure 1. Considered geometries: (a-f) three-dimensional, (g-j) two-dimensional, and (k) onedimensional. The direction of the applied field or force is given by $\boldsymbol{h}$.

The rich variety of phenomena leads to question how Bruggeman-type descriptions can be rationalized in a transparent manner. How many parameters are necessary for the description of the above-mentioned geometries and physical phenomena? Other questions are how effective-field theories are affected by nanoscale effects, and whether and how one can generalize them to describe multiferroic phenomena, which simultaneously involve magnetic, dielectric, and elastic degrees of freedom.

\section{GENERALIZED BRUGGEMAN THEORY}

Bruggeman's approach considers two phases characterized by materials constants $A_{\mathrm{I}}$ and $A_{\mathrm{II}}$, and volume fractions $\varphi_{\mathrm{I}}$ and $\varphi_{\mathrm{II}}$, respectively. For convenience, we write $\varphi_{\mathrm{II}}=$ $\varphi$ and $\varphi_{\mathrm{I}}=1-\varphi$. When the volume fraction of the second phase is small, then linearization yields $A_{\text {eff }}=A_{\mathrm{I}}\left(1+f\left(A_{\mathrm{I}}, A_{\mathrm{II}}\right) \varphi\right)$. The material is then described by an effective materials constant $A_{\text {eff }}=A_{\text {eff }}\left(A_{\mathrm{I}}, A_{\mathrm{II}}, \varphi\right)$. For a variety of specific systems, the function $f\left(A_{\mathrm{I}}, A_{\mathrm{II}}\right)$ has been determined by explicit calculation. We will discuss physical systems below, but the aim of this section is a derivation of $f$ without reference to particular composites.

\section{$\underline{\text { Parameterization }}$}

The function $f$ can often be written as $f\left(A_{\mathrm{I}}, A_{\mathrm{II}}\right)=\left(\alpha A_{\mathrm{I}}+\beta A_{\mathrm{II}}\right) /\left(\gamma A_{\mathrm{I}}+\delta A_{\mathrm{II}}\right)$, so that 


$$
A_{\mathrm{eff}}=A_{\mathrm{I}}\left(1+\frac{\alpha A_{\mathrm{II}}+\beta A_{\mathrm{I}}}{\gamma A_{\mathrm{II}}+\delta A_{\mathrm{I}}} \phi\right)
$$

For example, in the dielectric case, the susceptibility enhancement due a spherical inclusion of susceptibility $\varepsilon_{I I}$ is

$$
\varepsilon_{\mathrm{eff}}=\varepsilon_{I}\left(1+\phi \frac{3\left(\varepsilon_{I I}-\varepsilon_{I}\right)}{\varepsilon_{I I}+2 \varepsilon_{I}}\right)
$$

This means that $\alpha=3, \beta=-3, \gamma=1$, and $\delta=2$ for the dielectric response of spherical inclusions. However, these values are not universal but depend on the physical phenomenon and on the geometry and dimensionality of the composite.

Equation (1) contains the physics of the considered composite and forms the starting point for Bruggeman's selfconsistent effective-medium theory. Before applying the selfconsistent calculation to the general equation (1) we will first investigate whether the four parameter $\alpha, \beta, \gamma$, and $\delta$ are all independent. In fact, based on general considerations, without reference to any specific physical system, we can simplify equation (1).

For nearly homogeneous composites, where $A_{\mathrm{II}} \approx A_{\mathrm{I}}$, then $A_{\mathrm{eff}}$ is equal to the volume average of the two phases, $A_{\text {eff }}=(1-\phi) A_{\mathrm{I}}+\phi A_{\mathrm{II}}$. Evaluating this limit in Eq. (1) yields $\alpha=\gamma+\delta, \beta=-(\gamma+\delta)$, and

$$
A_{\mathrm{eff}}=A_{\mathrm{I}}\left(1+\phi \frac{A_{\mathrm{II}}-A_{\mathrm{I}}}{g A_{\mathrm{II}}+(1-g) A_{\mathrm{I}}}\right)
$$

where $g=\mathcal{H}(\gamma+\delta)$. In other words, in Eq. (1) there is only one independent parameter. For example, Eq. (2) yields $g=1 / 3$ for the susceptibility modification due to embedded spheres.

\section{Selfconsistency}

Figure 2 illustrates the selfconsistent embedding procedure used in Bruggeman's approach. Rather than embedding phase II (black) in phase I (white), or vice versa, both phases are embedded in an effective medium (gray). Physically, the neighborhood of a phase-II region is a mixture of phases I and II, rather than the pure matrix phase. As indicated in Fig. 4, this embedding is done for both phase, because regions of the matrix (phase I) have a mixed neighborhood, too. Explicitly,

and

$$
A_{\mathrm{eff}, \mathrm{I}}=A_{\mathrm{eff}}\left(1+\phi_{\mathrm{o}} \frac{A_{\mathrm{I}}-A_{\mathrm{eff}}}{g A_{\mathrm{I}}+(1-g) A_{\mathrm{eff}}}\right)
$$

$$
A_{\text {eff,II }}=A_{\text {eff }}\left(1+\phi_{\mathrm{o}} \frac{A_{\mathrm{II}}-A_{\mathrm{eff}}}{g A_{\mathrm{II}}+(1-g) A_{\mathrm{eff}}}\right)
$$




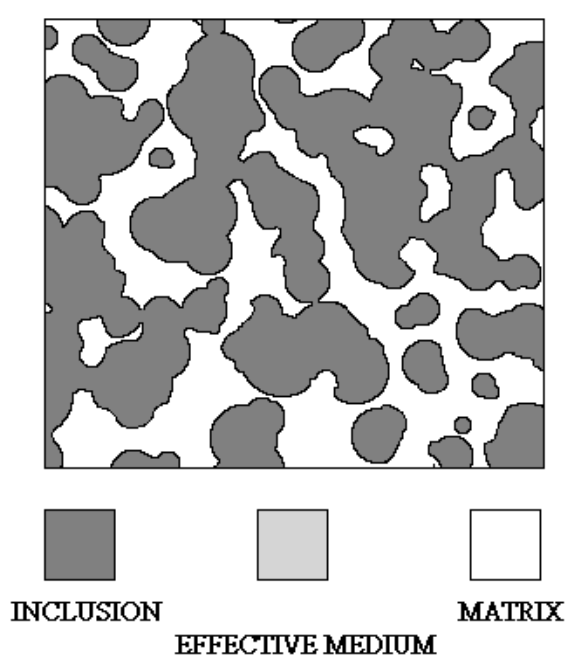

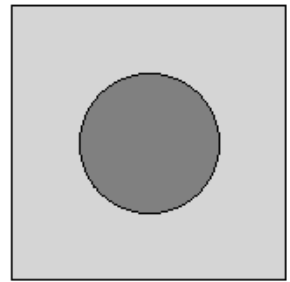

Weight: $\phi$

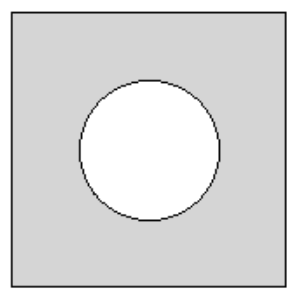

Weight: $1-\phi$

Figure 2. Real environment (schematic) and selfconsistent embedding in an effective medium.

where the fictitious volume fraction $\phi_{\mathrm{o}}$ will not enter the final result. Next, $A_{\mathrm{eff}}$ is obtained by averaging over the two configurations, that is, $A_{\text {eff }}=(1-\phi) A_{\text {eff,I }}+\phi A_{\text {eff,II. This yields }}$

$$
A_{\mathrm{eff}}=\phi A_{\mathrm{eff}}\left(1+\frac{A_{\mathrm{II}}-A_{\mathrm{eff}}}{g A_{\mathrm{II}}+(1-g) A_{\mathrm{eff}}} \phi_{\mathrm{o}}\right)+(1-\phi) A_{\mathrm{eff}}\left(1+\frac{A_{\mathrm{I}}-A_{\mathrm{eff}}}{g A_{\mathrm{I}}+(1-g) A_{\mathrm{eff}}} \phi_{\mathrm{o}}\right)
$$

and

$$
\phi \frac{A_{\mathrm{II}}-A_{\mathrm{eff}}}{g A_{\mathrm{II}}+(1-g) A_{\mathrm{eff}}}+(1-\phi) \frac{A_{\mathrm{I}}-A_{\mathrm{eff}}}{g A_{\mathrm{I}}+(1-g) A_{\mathrm{eff}}}=0
$$

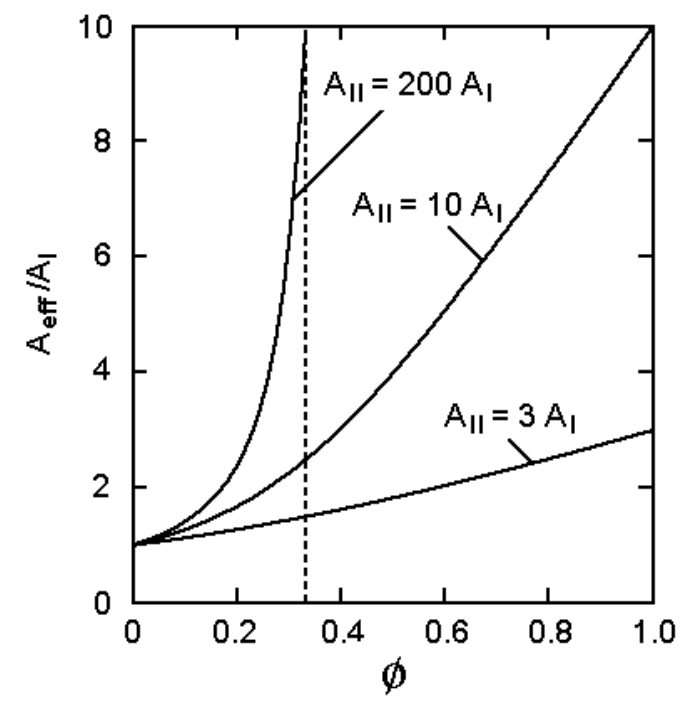

Figure 3. Response as a function of volume fraction. 
Equation (6) is, essentially, a quadratic equation for $A_{\text {eff. }}$ Aside from the sign of the physically relevant solution, which must be determined separately,

$$
A_{\mathrm{eff}}=\frac{A_{\mathrm{II}}(\phi-g)+A_{\mathrm{I}}(1-g-\phi)}{2(1-g)}\left(1 \pm \sqrt{1+\frac{4 A_{\mathrm{II}} A_{\mathrm{I}} g(1-g)}{\left(A_{\mathrm{II}}(\phi-g)+A_{\mathrm{I}}(1-g-\phi)\right)^{2}}}\right)
$$

Figure 3 shows some typical $A_{\text {eff }}(\phi)$ curves for $g=1 / 3$.

\section{$\underline{\text { Percolation }}$}

When $A_{\text {II }}$ goes to zero or to infinity, then Eq. (7) yields a percolation transition. For example, metallic regions in an insulator do not affect the resistivity until the metallic volume fraction reaches the percolation threshold $\phi=\phi_{c}$, and a sol-gel transition is accompanied by a divergence of the viscosity. The dashed line in Fig. 3 illustrates percolation at $\phi_{\mathrm{c}}=1 / 3$.

Putting $A_{\mathrm{II}}=0$ and $A_{\text {eff }}=0$ in Eq. (6) yields the percolation threshold $\phi_{\mathrm{c}}=1-\mathrm{g}$, whereas $A_{\mathrm{II}}=\infty$ and $A_{\text {eff }}=\infty$ leads to $\phi_{\mathrm{c}}=g$ and $A_{\text {eff }}=A_{\mathrm{I}} /\left(1-\phi / \phi_{\mathrm{c}}\right)$. The latter relation is a power-law with a dimensionality-independent mean-field exponent. This is not surprising, because the assumption of an effective field (Fig. 2) is similar to the meanfield approximation in magnetism. Each region of the composite interacts with an average environment only, but the percolating backbone is a long-range phenomenon and very susceptible to fluctuations caused by structural randomness [12]. As a consequence, the present approach is not able to provide an accurate description of structures such as that shown in Fig. 3, even if the percolation threshold is $\phi_{\mathrm{c}}$ is close to the exact value.

\section{Conjugate Relations}

Equations (1) and (3) describe a rich variety of composite materials constant. In general, these constants from pairs, depending on how they link the applied force or field $h$ (or $\Xi$ ) to the response m. In general, the energy of the material can be written as $H=V$ $\mathrm{mh}$, corresponding to the material equation $\partial H / \partial m=0$, or $\partial U / \partial m=f$. Neglecting nonlinear terms, $U=1 / 2 G m^{2}$ and $h=G m$. Since $h$ and $m$ are conjugate variables, Legendre transformation yields $U=1 / 2 h^{2} / G+m h, H=h^{2} / 2 G, \partial H / \partial f=-m$, and $J h=m$, where $J=1 / G$. In mechanical systems, $G$ and $J$ are known as stiffness and compliance, respectively. Examples of $G$ and $J$ are Young's modulus and the tensile compliance, respectively [13]. Evaluating Eq. (3) for $A=G$ and $A=J=1 / G$ reveals that going from $G$ to $J$, or vice versa, reduces to the simple transformation $g \rightarrow 1-g$. Since $G=\infty$ corresponds to $J=0$, and $G=0$ to $J=\infty$, this transformation relates percolation-type singularities of conjugate properties, meaning the sum of the two thresholds is equal to one.

\section{The Parameter $g$}

To determine the parameter g, it is necessary to specify Eq. (3) for the considered type of composite. In general, this can only be done by explicit calculation, that is, partial 
differential equation that describing the local response $m(\mathbf{r})[3-7,14]$. We will briefly outline the calculation for phenomena described by $\nabla^{2} \Phi=0$, where $\Phi$ is a potential whose gradient is a local field. Examples are magnetostatic and dielectric phenomena and diffusion processes. The differential equation is subject to appropriate boundary conditions. Far away from the inclusion implied by Eqs. (1) and (3), the system is homogeneous, so that $\nabla \Phi(\mathbf{r})$ is constant, whereas at interfaces, the parallel force and perpendicular flux components are conserved. In magnetic systems, this refers to $\boldsymbol{H}$ and $\boldsymbol{B}$, respectively, whereas the respective diffusion quantities are the concentration gradient and the particle flux. Figure 4 shows the flux lines for an inclusion. In the diffusion analogy, (a) shows an inclusion with a high diffusivity, so that the inclusions attracts flux lines, whereas (b) shows an inclusion with low diffusivity, which repels flux lines.

The calculation for spherical geometries, Fig. 1(a), shows that the parameter $g$ is equal to $1 / 3$. More generally, both $g$ and $\phi_{\mathrm{c}}$ are equal to the demagnetizing factor for the considered inclusion geometry. This is a direct consequence of the boundary conditions but has also a transparent physical meaning (see below). However, other scenarios may yield different relationships. For example, in mechanical systems, there are different stress and strain scenario, and the behavior depends on Posisson's ratio [7].

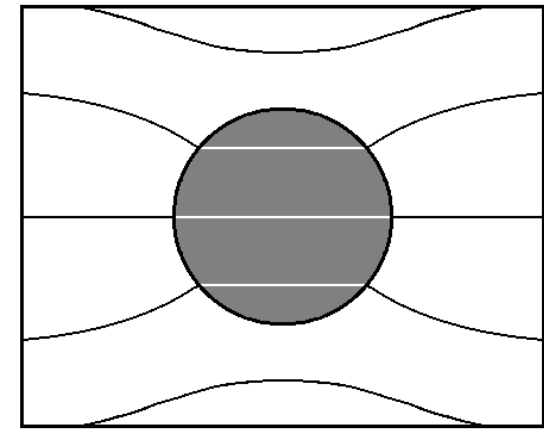

(a)

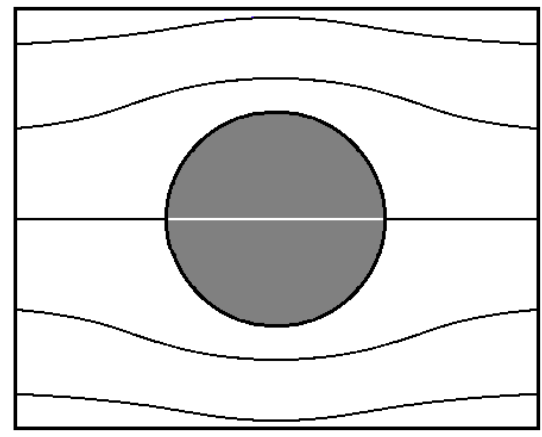

(b)

Figure 4. Flux-line modification due to inhomogenities: (a) flux-line attraction in regions with large compliance and (b) flux-line repulsion in regions with small compliance.

\section{$\underline{\text { Geometry and Dimensionality }}$}

The parameter $g$ depends on the geometry and dimensionality of the considered geometry (Fig. 1). In general, the determination of $g$ requires explicit calculation, as indicated in Fig. 4. However, for systems with very high symmetry, as in Fig. 1(a), (b), (h), (j), and (k), solutions are easily obtained. For example, Young's modulus $E$ for a fiber-reinforced polymer of geometry (a) has the percolation threshold $\phi_{\mathrm{c}}=0$, because a single fiber of infinite stiffness is sufficient to ensure zero tensile strain. As a consequence, $g=0$, and $E_{\text {eff }}=(1-\phi) E_{\mathrm{I}}+\phi E_{\mathrm{II}}$. The same applies to the geometry of Fig. 1(h).

Another simple example is the onset of conductivity due to a metallic phase embedded in an insulator. In the geometry of Fig. $1(\mathrm{k}), \phi_{\mathrm{c}}=1$, so that $g=1$. Exploiting that $g \rightarrow 1-g$ for conjugate problems, we obtain the resistivity $\rho_{\text {eff }}=(1-\phi) \rho_{\mathrm{I}}+\phi \rho_{\mathrm{II}}$. 
This is easy to understand, because the considered configuration is essentially a series of resistors. Similarly, the geometries (a) and (h) have the character of parallel resistors, and the conductivity obeys $\sigma_{\text {eff }}=(1-\phi) \sigma_{\mathrm{I}}+\phi \sigma_{\mathrm{II}}$. In mechanics, effective materials constants for series and parallel geometries are known as Reuss and Voigt (or Kelvin) averages [13], so that $g=1$ and $g=0$ can be interpreted as Reuss and Voigt response parameters, respectively.

For complicated inclusion geometries, it is generally difficult to determine g. An exception are systems described by $\nabla^{2} \Phi=0$ and subject to the boundary conditions of the previous subsection. This includes many magnetic, dielectric, and transport composites but does not apply to mechanical composites, where Poisson's ratio $v$ is an additional consideration. In terms of the magnetostatic analogy, $g=D_{\text {mag }}$, where $D_{\text {mag }}$ is the demagnetizing factor of the inclusion. As a consequence, $g=1 / 3$ for spherical inclusions, Fig. 1(c), and $g=1 / 2$ for wires embedded perpendicular to the field $\mathrm{h}$, as in Fig. 1(f). Demagnetizing factors for other geometries can be found, for example, in [15].

For d-dimensional hyperspheres, Figs. $1(\mathrm{c}),(\mathrm{g})$, and $(\mathrm{k}), g=1 / d$, where $d$ is the dimensionality of the matrix. Note that $g=1 / 2$ for the geometries (f) and (g), so that both the compliance and the stiffness constants have the same percolation threshold. This duality is typical of two-dimensional percolation phenomena. The site-percolation for a three-dimensional cubic lattice, $\phi_{c}=0.3116$ [12], is very close to $1 / 3$ but somewhat accidental, because $\phi_{c}$ exhibits a pronounced dependence on details of the geometry.

\section{$\underline{\text { Static and Dynamic Properties }}$}

The time-dependent response to a external force or field, which gives rise to viscoelastic behavior in mechanical composites and to frequency-dependent losses, is often described or well approximated the material's equation

$$
h=G m+\eta \mathrm{dm} / \mathrm{dt}
$$

Here $\eta$ is a generalized viscosity [13]. This equation describes relaxation processes in terms of the relaxation time $\tau=\eta / G$. Fourier transformation yields the complex equation $h^{*}=G^{*} m^{*}$, where $G^{*}=G+\mathrm{i} \omega \eta$. Equating $G^{*}$ with $A$ yields, from Eq. (7), the effective stiffnesses and viscosities as real and imaginary parts of $A_{\text {eff, }}$ respectively. The same applies to $J^{*}=1 / G^{*}$.

In the mechanical analogy, Eq. (8) means $\sigma=G \varepsilon+\eta \mathrm{d} \varepsilon / \mathrm{d} t$, and $G$ and $h$ are the shear modulus and the mechanical viscosity, respectively. Transport properties correspond to $G=\infty$ in Eq. (8). Examples are diffusivity, thermal conductivity, and-as conjugate properties - conductivity and resistivity. 


\section{CASE STUDIES}

\section{$\underline{\text { Mechanical Behavior }}$}

Parameters $g$ for various elastic, viscous, and viscoelastic properties easily obtained from continuum calculations. Comparing Eq. (3) for [3,4,7,16]. For spherical inclusions, the shear modulus $G$ and the bulk modulus $K$ are described by

$$
g=\frac{2\left(4-5 v_{\mathrm{o}}\right)}{15\left(1-v_{\mathrm{o}}\right)} \quad \text { and } \quad g=\frac{1+v_{\mathrm{o}}}{3\left(1-v_{\mathrm{o}}\right)}
$$

respectively. In both equations, $v_{\mathrm{o}}$ is Poisson's ratio of the matrix, so that conjugate percolation thresholds may not add up to one. When the material as incompressible, $v_{\mathrm{o}}=$ $1 / 2$ and $K=\infty$, the shear modulus is described by $g=2 / 5$. In a very good approximation, this is realized for reinforced rubber composites, which are used, for example, in car tires $[17,18]$.

Since the complex viscoelastic modulus is given by $G^{*}=G+\mathrm{i} \omega \eta$, the incompressive limit includes the viscosity enhancement due to particles suspended in a liquid. For rigid spheres, $G_{\mathrm{II}}=\infty$, the viscosity enhancement reduces to Einstein's famous formula $\eta=\eta_{\mathrm{o}}(1+2.5 \phi)[3,4,7,16]$. Since $g=2 / 5$, percolation is predicted when the volume fraction of the hard phase reaches $40 \%$. This is close to the experimental value of slightly more than $45 \%$ [7].

\section{Electric and Magnetic Phenomena}

The dielectric and magnetic polarization obey $D=\varepsilon E$ and $M=\chi H$, respectively. In three-dimensional systems, the compliances $\varepsilon$ and $\chi$ are described by $g=D_{\text {mag }}$, whereas hyperspherical inclusions lead to $g=1 / d$. In a strict sense these equations, are limited to the linear regime, where the polarization is small. In ferromagnetic and ferroelectric systems, it is important to consider nonlinear terms, nanoscale effects, and mixing electric, magnetic, and elastic terms (see below).

Dynamic phenomena such as damping and line-width broadening are included by considering complex quantities $\varepsilon^{\prime \prime}=\varepsilon^{\prime}+\mathrm{i} \varepsilon^{\prime \prime}$ and $\chi=\chi+\mathrm{i} \chi^{\prime \prime}$. Since both the static dielectric polarization and the conductivity are caused by a displacement of charged particles (electrons), $\varepsilon^{*}$ accounts for both phenomena, and $\varepsilon^{\prime \prime}$ is, essentially, the conductivity. Magnetic relaxation is only partly described by $\chi^{\prime \prime}$, because the slow or 'magnetic viscosity' dynamics $M(t)$ of magnetic materials reflects thermally activated jumps over energy barriers rather than linear viscosity $[15,19]$.

\section{$\underline{\text { Transport Properties }}$}

Transport amounts to a flux $\boldsymbol{j}$ subject to particle-number, charge, or energy conservation, $\nabla \cdot \boldsymbol{j}=0$. The flux is driven by an external force, $\boldsymbol{j}=\boldsymbol{\sigma} \boldsymbol{h}$, where $\sigma$ is a generalized conductivity [20]. (Essentially, $\sigma$ is the imaginary part of the complex compliance.) 
These relations, which are equivalent to the electrostatic equations $\nabla \cdot \boldsymbol{D}=0$ and $\boldsymbol{D}=\boldsymbol{\varepsilon} \boldsymbol{E}$, and the fact that $\boldsymbol{h}$ and $\boldsymbol{E}$ are gradients of a potential $\boldsymbol{\Phi}$, mean that the transport amounts to $g=D_{\text {mag. }}$. For example, embedding spherical particles of in a matrix is described by $g=$ 1/3, whereas parallel and series geometries, such as in Fig. 1(a) and (b), imply $g=0$ and $g$ $=1$, respectively.

One phenomenon described by this relationship is electrical conduction, as described by the conductivity $\sigma$, or resistivity $\rho=1 / \sigma$. In the sense of Eq. (8), these quantities are related by $\varepsilon^{*}=\varepsilon$ - i $\sigma \omega$ and $\sigma^{*}=\sigma+\mathrm{i} \omega \varepsilon$. Some other phenomena are heat conduction and chemical diffusion, where the relation between force and flux density is given by the respective Fick's laws $j=-D \nabla \cdot c$ and $j=-\lambda \nabla T$.

\section{NANOSCALE AND MULTIFERROIC EFFECTS}

\section{$\underline{\text { Nanoscale interactions }}$}

A key feature of the 'macroscopic' Bruggeman systems considered above is that they do not involve a characteristic length scale. This is a consequence of the underlying partial differential equation $\nabla^{2} \Phi=0$. However, many systems encountered in practice are described by equations of the type $\nabla^{2} \Phi+\kappa \Phi=0$, where $\kappa$ is an inverse screening length $[19,21]$. Examples are nanoscale magnetic systems, Debye-Hückel electrolytes, and Landau-Ginzburg type systems. The screening length reflect the competition between different local and non-local energy contributions and is often of the order of a few nanometer.

Figure 5 illustrates this screening effect by comparing Coulomb $(\kappa=0)$ and Yukawa $(\kappa>0)$ solutions for a spherical geometry. (The Yukawa theory was originally introduced to discuss the difference between short-range nuclear and long-range Coulomb interactions.) For example, the behavior of ferromagnetic two-phase composites is governed by an exchange length proportional to the square root of the exchange stiffness $A$ and magnetic anisotropy $K_{1}$.

This has far-reaching consequences for the understanding of advanced mechanical and electromagnetic composites, because the structural correlation length (inclusion size) tends to interfere with the length scale of the interaction. Consider, for example, a composite where hard-magnetic spheres $\left(K_{1}=\infty\right)$ are embedded in a soft magnetic matrix of anisotropy $K_{\mathrm{s}}=\mu_{0} H_{\mathrm{s}} M_{\mathrm{s}} / 2$ This regime has been considered for energy-product enhanced two-phase magnets $[9,19,22]$. Figure 6 shows the geometry and the corresponding effective-field model. The magnetization of the hard inclusions is assumed to point up, and the appropriate boundary condition for the small 'perpendicular' magnetization component $\mathrm{m}$ is condition $\partial m /\left.\partial r\right|_{r=L}=0$. The calculation of the nucleationfield coercivity is similar to that in $[9,21]$ and yields, for small volumes,

$$
H_{\mathrm{c}}=H_{\mathrm{s}}+6 A \phi / \mu_{0} \mathrm{M}_{\mathrm{s}} R^{2}
$$


This means that $g$ is a function of the radius $R$ of the inclusions rather than a constant. Physically, the hard-magnetic spheres create an 'interaction cloud' of a exchange-coupled soft-magnetic material. This cloud also affects the percolation transition.

A similar effect is rubber reinforcement, where small inclusions such as iron oxides, silica, and carbon black (soot) improve the mechanical properties of the material. For large particles, one can use the Einstein formula, $E=E_{\mathrm{o}}(1+2.5 \phi)$, but highperformance fillers have particle radii $R$ of about $10 \mathrm{~nm}$, which is comparable to the average end-to-end distance $R_{\mathrm{N}} \sim N^{1 / 2}$ of the polymer chains [17,18,23]. The stiffness of cross-linked polymer networks obeys $E \sim n k_{\mathrm{B}} T$, where $\mathrm{n}$ is the cross-link density. Since the surface of the particle creates additional crosslinks, there is an additional contribution roughly proportional to the number of chains that touch a particle [17]. This means that $g$ is a function of $R_{\mathrm{N}} / R$.

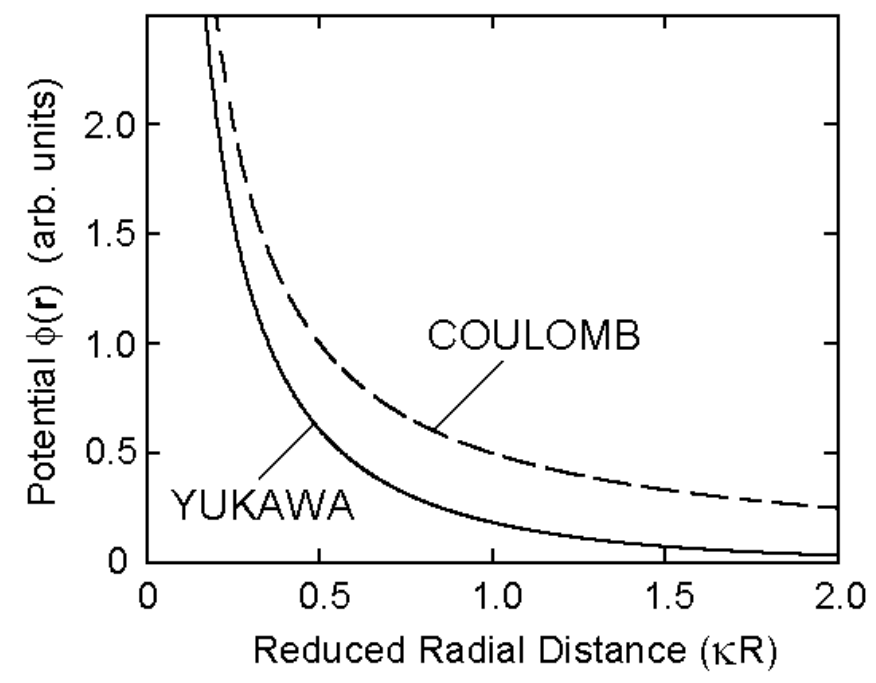

Figure 5. Range of interactions. In solids and liquids, both long-range power-law (e.g. Coulomb) and finite-range exponential interactions occur.

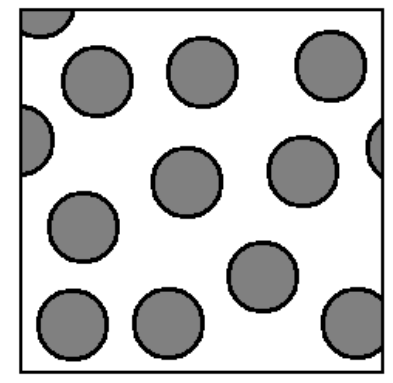

(a)

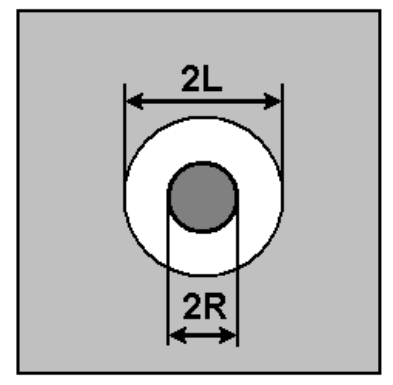

(b)

Figure 6. Nanoscale effects: (a) well-textured magnetically hard grains in a soft-magnetic or semihard matrix and (b) effective-field description. The response of the material depends not only on the volume fraction $\Phi=R^{3} / L^{3}$ but also, in a separate manner, on $R$. 


\section{$\underline{\text { Multiferroic Effects }}$}

Multiferroic composites are of interest as sensors and for microwave, optical, and other applications. They are characterized by the simultaneous exploitation of magnetic, electric and other degrees of freedom (order parameters). The Bruggeman description of these structures depends on whether the interactions between different degrees of freedom are local or nonlocal [11]. In the most general case, nonlocal interactions (between, for example, magnetic and electric degrees of freedom) lead to a complicated k-dependent mixture of electric and magnetic polarizations. In addition, nonlinear effects are important in most multiferroic systems. For example, when considering multifunctional materials with interactions involving two or more physical properties, such as magnetoelastic coupling, the parameter $g$ generally describes a mixture of materials constants.

However, in most cases of practical interest it is possible to simplify the problem. For example, insulating magnetic particles embedded in a dielectric matrix tend to be electrically inert, so that $\varepsilon(\mathbf{r})=\varepsilon_{\mathrm{o}}$ [24]. In addition, it is often possible to treat interactions and nanoscale effects as small perturbations, which makes the systems easier to describe.

\section{SUMMARY AND CONCLUSIONS}

Table I summarizes the effects treated in this paper. As outlined above, the response parameter $g$ depends on the considered physical phenomenon and on the geometry and dimensionality of the composite. In many cases, there exist simple relationships between the original materials parameter and the corresponding reciprocal (inverse) parameter, for example modules and compliance (or resistivity and conductivity). Phenomena such as diffusion, heat conduction and dielectric response in three-dimensional composites are described by $g=D_{m a g}$. For mechanical constants, such as $G, K$, and $E(G, K)$, the situation is slightly more complicated, Eq. (9).

Table I. Bruggeman response of various materials. For simplicity, the response parameters quoted in this table are those for inclusions embedded in a three-dimensional matrix.

\begin{tabular}{|c|c|c|c|c|c|c|}
\hline System & $\begin{array}{l}\text { Force } \\
\text { (h) }\end{array}$ & $\begin{array}{l}\text { Response } \\
\text { (m) }\end{array}$ & $\begin{array}{l}\text { Stiffness } \\
(G)\end{array}$ & $\begin{array}{l}\text { Compliance } \\
(J)\end{array}$ & $\begin{array}{l}\text { Parameter } \\
\text { (g) }\end{array}$ & $\begin{array}{l}\text { Potential } \\
(\Phi)\end{array}$ \\
\hline Dielectric & $E$ & $D$ & $1 / \varepsilon$ & $\varepsilon$ & $D_{\mathrm{mag}}$ & $\phi_{\mathrm{el}}$ \\
\hline Conductive & $E$ & $j$ & $\rho$ & $\sigma$ & $D_{\text {mag }}$ & $\phi_{\mathrm{el}}$ \\
\hline Magnetic & $H$ & $M$ & $1 / \chi$ & $\chi$ & $D_{\text {mag }}$ & $\phi_{\mathrm{mag}}$ \\
\hline Diffusive & $\nabla c$ & $j$ & $1 / D_{\text {iff }}$ & $D_{\text {diff }}$ & $D_{\text {mag }}$ & $c$ \\
\hline Thermal & $\nabla T$ & $\mathrm{j}$ & $1 / \lambda$ & $\lambda$ & $D_{\text {mag }}$ & $T$ \\
\hline Elastic & $\sigma($ stress $)$ & $\mathcal{E}($ strain $)$ & $G, \mathrm{~K}, E$ & $J$ & $\mathrm{~g}\left(v_{\mathrm{o}}\right)$ & $\phi_{\text {mech }}$ \\
\hline Viscous & $\sigma($ stress $)$ & $\mathcal{E}($ strain $)$ & $\eta$ & 1 & $2 / 5$ & $\phi_{\text {mech }}$ \\
\hline
\end{tabular}


In conclusion, we have elaborated a generalized Bruggeman theory and discussed its applicability. The theory describes mechanical, transport, dielectric, and magnetic composites for a wide range of volume fractions. It's a single-parameter theory, characterized by a response parameter $g$ that is equal to the percolation threshold. However, the long-range critical correlation are ignored, and the percolation behavior is mean-field like. When nanoscale, nonlinear and multiferroic effects are involved, the theory must be modified, and $g$ depends on additional parameters, such as the particle size. - This work is supported by NSF-MRSEC, the W. M. Keck foundation, AFOSR, and ARO.

\section{REFERENCES}

[1] D. A. G. Bruggeman, Ann. Phys. (5) 24, 637 (1935).

[2] E. Madelung and S. Flügge, Ann. Phys. (5) 22, 209 (1935).

[3] A. Einstein, Ann. Phys. 17, 549 (1905).

[4] A. Einstein, Ann. Phys. 19, 289 (1906).

[5] Z. Hashin, J. Appl. Mech. 29, 143 (1962).

[6] Z. Hashin and S. Shtrikman, J. Appl. Phys. 33, 3125 (1962).

[7] R. M. Christensen, Mechanics of Composite Materials, (Wiley, New York, 1979).

[8] R. Skomski and J. M. D. Coey, Phys. Rev. B 48, 15812 (1993).

[9] R. Skomski and J. M. D. Coey, IEEE Trans. Magn. 30, 607 (1994).

[10] W. T. Doyle, J. Appl. Phys. 85, 2323 (1999).

[11] A. Chipara, D. Hul, J. Sankar, D. Leslie-Pelecky, A. Bender, L. Yue, R. Skomski, and D. J. Sellmyer, Composites Part B-Engineering 35, 235 (2004).

[12] D. Stauffer and A. Aharony, Introduction to Percolation Theory, (Taylor \& Francis, London 1992).

[13] I. M. Ward and D. W. Hadley, Mechanical Properties of Solid Polymers, (Wiley, New York, 1993).

[14] J. M. Dewey, J. Appl. Phys. 18, 578 (1947).

[15] R. Skomski and J. M. D. Coey, Permanent Magnetism, (Institute of Physics, Bristol, 1999).

[16] T. S. Chow, Mesoscopic Physics of Complex Materials, (Springer, New York, 2000).

[17] R. Skomski, Diplomarbeit, THLM Leuna-Merseburg (1986).

[18] B. Erman and J. E. Mark, Structures and Properties of Rubberlike Networks, (Oxford University Press, New York, 1997).

[19] R. Skomski, J. Phys.: Condens. Matter 15, R841 (2003).

[20] H. C. Berg, Random Walks in Biology, (Princeton University Press, Princeton, New Jersey, 1993).

[21] R. Skomski. J. Magn. Magn. Mater. 272-276, 1476 (2004).

[22] A. E. Berkowitz, M. F. Hansen, K. S. Vecchio, F. T. Parker, H. Harper, and F. E. Spada, in: Rare Earth Magnets and Their Applications, Eds. G. C. Hadjipanayis and M. J. Bonder, (Rinton Press, Princeton, 2002) p. 749-759.

[23] G. Heinrich, E. Straube, and G. Helmis, Adv. Polym. Sci. 85, 33 (1988).

[24] J.Y. Li, Phys. Rev. Lett. 90, 217601 (2003). 